\title{
Les précurseurs des protamines dans le spermatozoïde humain
}

\author{
P Chevaillier 1, A Martinage 2, A Arkhis 1, P Sautière 2 \\ 1 Université Paris-Val de Marne, laboratoire de biologie cellulaire, 94010 Créteil; \\ 2 Institut de recherches sur le cancer, URA CNRS $n^{\circ}$ 409, 59045 Lille, France
}

(15 réunion du groupe Développement INRA, Paris, 24-26 mai 1989)

\begin{abstract}
Résumé - Le noyau du spermatozoïde humain est caractérisé par un équipement en protéines basiques très hétérogène. Trois groupes de protéines ont en effet été isolés : des histones de type somatique ainsi que des variants spécifiques du testicule, des protamines, et des protéines à mobilité électrophorétique intermédiaire entre celle des histones et celle des protamines. Les protamines humaines se répartissent en 2 familles de protéines de structures différentes. Les protamines HP1 diffèrent entre elles par leur degré de phosphorylation; les protamines HP2, 3 et 4 diffèrent par leur séquence $\mathrm{N}$-terminale. Les proténes basiques intermédiaires (HPI1, HPI2, HPS1, HPS2) possèdent une région C-terminale commune de 54 résidus, identique à la séquence de la protamine HP3. Seules leurs séquences $\mathrm{N}$-terminales diffèrent d'une protéine à l'autre. Compte tenu de ces parentés structurales, la protéine intermédiaire HPI1 apparaît comme un précurseur probable des protamines HP2 et HP3.
\end{abstract}

protamine / chromatine / spermatozoïde / homme

Summary - Molecular characterization of precursors for human sperm protamines. Basic proteins isolated from human sperm nuclei are highly heterogeneous. Three groups of nuclear basic proteins have been characterized: somatic-type as well as testis-specific histones, protamines and basic proteins with an electrophoretic mobility which is intermediate between that of histones and that of protamines. Human protamines can be separated into 2 protein families with different amino acid composition and amino-acid sequence. Protamines HP1 differ in their degree of phosphorylation. Protamines HP2, 3 and 4 differ by their amino-terminal sequence. Intermediate basic proteins (HPI1, HPI2, HPS1, HPS2) share a common C-terminal sequence of 54 residues identical to the amino-acid sequence of protamine HP3; only their N-terminal regions are different. Taking into account these structural homologies, the intermediate basic protein HPI1 appears as a precursor of protamines HP2 and HP3.

protamine / chromatin / sperm / human 


\section{INTRODUCTION}

Le noyau du spermatozoïde des Mammifères est généralement caractérisé par la présence d'une protamine, protéine très basique, de faible poids moléculaire, riche en arginine et en cystéine. Seuls la souris et l'étalon possèdent 2 types de protamines de structures différentes (Kleene et al, 1985; Bellvé et al, 1988; Bélaïche et al, 1987; Pirhonen et al, 1989). Dans tous les cas, les histones ont été éliminées des noyaux de spermatides en cours de différenciation.

L'équipement en protéines basiques du noyau du spermatozoïde humain s'est révélé très hétérogène. Trois groupes de protéines nucléaires basiques ont été isolés, dans un ordre de mobilité électrophorétique croissante : les histones, les protéines basiques intermédiaires et les protamines (Gusse et al, 1986), ces dernières étant les plus abondantes et se répartissant en 2 familles distinctes. Les protéines basiques intermédiaires présentent des caractères structuraux les rapprochant de l'une de ces 2 familles de protamines. Le présent article rapporte les conclusions que l'on peut tirer de la détermination de la structure primaire de ces protéines et la filiation qui peut exister entre elles.

\section{MATÉRIELS ET MÉTHODES}

Les protéines nucléaires sont extraites de noyaux purifiés à partir de spermes d'hommes normalement féconds ou ne présentant pas d'anomalie détectable au microscope photonique. En résumé, ces noyaux sont débarrassés de leur enveloppe nucléaire; les protéines sont réduites par le dithiothréitol, alkylées par liodoacétamide et extraites par l'HCl $0,25 \mathrm{~mol}^{-l^{-1}}$. Elles sont purifiées par chromatographie d'échange d'ions sur carboxyméthylcellulose et par HPLC en phase inverse. Les protamines et protéines intermédiaires sont analysées par électrophorèse sur gel de polyacrylamide en acide-urée; les histones sont de plus caractérisées par électrophorèse sur des gels contenant du SDS ou du Lubrol. Les méthodes utilisées pour la détermination de la composition en acides aminés et l'établissement des structures primaires des protéines ou des peptides obtenus par digestion enzymatique ont été détaillées dans les publications précédentes (Gusse et al, 1986; Sautière et al, 1988).

\section{RÉSULTATS ET DISCUSSION}

L'analyse électrophorétique des protéines acido-solubles sur gel de polyacrylamide montre la présence de 3 classes de protéines: des histones, des protamines et des protéines à mobilité électrophorétique intermédiaire (fig 1).

Analysée de manière comparative sur 3 types de gel de polyacrylamide, la fraction des histones s'est révélée constituée d'histones de type somatique $(\mathrm{H} 3, \mathrm{H} 4$ ainsi que des formes mono- et multi-acétylées; H2A en petite quantité) et d'un variant spécifique du testicule de I'histone H2B (TH2B) abondamment représenté. Les histones de type $\mathrm{H} 1$ sont, par contre, absentes ou peu représentées.

Les protamines se répartissent chez l'homme en 2 familles. La première famille (HP1) correspond à des protéines riches en arginine, cystéine et tyrosine (fig 2). La protamine HP1 se rattache aux autres protamines P1 dont la séquence a été déterminée chez d'autres Mammifères (McKay et al, 1985). L'hétérogénéité des protamines HP1, telle que la révèle l'analyse électrophorétique, s'explique par l'existence de formes non phosphorylée (HP1b) et phosphorylée (HP1a) (fig 1b). La seconde famille de protamines comprend 3 protéines (HP2, HP3, HP4) dont les séquences $\mathrm{N}$-terminales diffèrent : la protamine HP2 (57 résidus) ne diffère de la protamine HP3 (54 résidus) que par la 


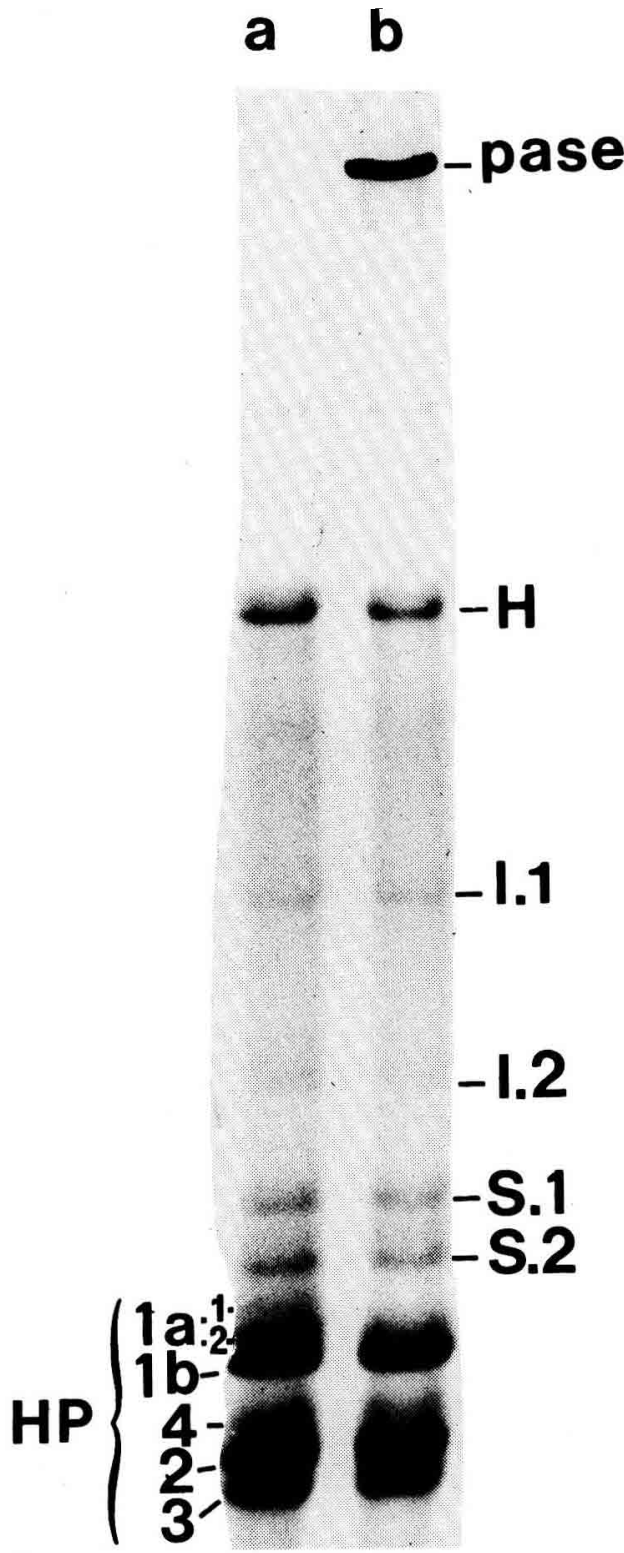

Fig 1. a) Analyse électrophorétique en gel de polyacrylamide acide-urée des protéines basiques totales du noyau du spermatozoïde humain; b) Protéines traitées par la phosphatase alcaline d'E coli (Pase). Noter la disparition des formes phosphorylées de la protamine HP1 (1a1,1a2). $H$ : histones; $11,12, S 1$, S2 : protéines basiques intermédiaires; HP1a, HP1b, HP2, HP3, HP4 : protamines. présence d'un tripeptide $\mathrm{N}$-terminal additionnel (McKay et al, 1986; Ammer ot al, 1986; Gusse et al, 1986). De plus, la protamine HP2 est présente en partie sous forme mono-phosphorylée.

Ces protamines se caractérisent par leur richesse en arginine, histidine et cystéine. La comparaison de leur structure primaire avec celle des autres protamines de Mammifères montre l'existence d'homologies de séquence avec les protamines mP2 de souris (Bellvé et al, 1988) et St2 d'étalon (Pirhonen et al, 1989) mais peu d'homologies avec les protamines de type P1.

Les protéines basiques intermédiaires (HPI1, HPI2, HPS1 et HPS2) qui persistent dans le noyau du spermatozoïde humain sont également des protéines riches en arginine, cystéine et histidine, et de plus elles renferment des quantités importantes quoique variables d'acide glutamique. L'établissement de la structure primaire de leur extrémité $\mathrm{N}$-terminale, alliée à la détermination de la composition en acides aminés de ces différentes protéines et de leurs cartes peptidiques obtenues par digestion avec l'endoprotéinase Lys-C et l'endoprotéinase Glu-C, a permis de conclure qu'il s'agissait d'une famille de protéines apparentées entre elles et aux protamines HP2 et HP3. Toutes ces protéines ont en commun une séquence d'acides aminés de 54 résidus qui représente leur région C-terminale et qui correspond à la protamine HP3. Compte tenu de ces parentés structurales, la protéine HPI1 peut être considérée comme le précurseur des protamines de type P2. Par ailleurs, la protéine HPI1 présente des homologies de structure importantes avec la protéine pmP2 de souris dont la structure a été établie à partir de l'ADNc correspondant (Yelick et al, 1987). Chez l'homme et chez la souris, les protamines de type P2 présentes dans le sperme dériveraient par 
HP 1 1 50

HP1 ARYRCCRSQSRSRYYRQRQRSRRRRRRSCQTRRRAMRCCRPRYRPRCRRH

HP 3 l
GQSHYRRRHCSRRR LHR I HRRQHRSCRRRKRRSCRHRRRHRRGCRTRKRTCRRH

HPI 1 1 VRYRVRSLSERSHEVYRQQLHGQEQGHHGQEEQGL . . . . .

HPI 2 1 HGQEQGHHGQEEQGL. . . . . .

HPS 1

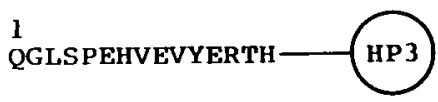

HPS 2

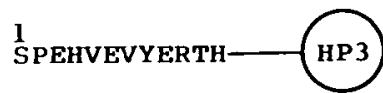

HP2

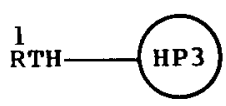

Fig 2. Structure primaire des protamines et des protéines basiques intermédiaires du spermatozoïde humain. Pour ces dernières, seules les parties $\mathrm{N}$-terminales ont été explicitées, les régions $\mathrm{C}$ terminales étant identiques à la séquence de la protamine HP3.

protéolyse limitée d'une pro-protamine. Lẹs systèmes enzymatiques impliqués ainsi que les modalités de cette maturation - ordonnée ou non - restent à préciser.

\section{RÉFÉRENCES}

Ammer H, Henschen A, Lee CH (1986) Isolation and amino-acid sequence analysis of human sperm protamines P1 and P2. Occurrence of two forms of protamine P2. Biol Chem Hoppo-Seyler 367, 515-522
Bélaïche D, Loir M, Kruggle W, Sautière P (1987) Isolation and characterization of two protamines St1 and St2 from stallion spermatozoa, and amino-acid sequence of the major protamine St1. Biochim Biophys Acta 913, 145-149

Bellvé AR, McKay DJ, Renaux BS, Dixon GH (1988) Purification and characterization of mouse protamines P1 and P2. Amino-acid sequence of P2. Biochemistry 27, 2890-2897

Gusse $M$, Sautière $P$, Bélaïche $D$, Martinage $A$, Roux C, Dadoune JP, Chevaillier P (1986) Purification and characterization of nuclear basic proteins of human sperm. Biochim Biophys Acta 884, 124-134 
Kleen KC, Distel RJ, Hecht NB (1985) Nucleotide sequence of a CDNA clone encoding mouse protamine $\mathrm{P} 1$. Biochemistry 24, 719722

McKay DJ, Renaux BS, Dixon GH (1985) The amino-acid sequence of human sperm protamine P1. Biosci Rep 5, 383-391

McKay DJ, Renaux BS, Dixon GH (1986) Human sperm protamines. Amino-acid sequences of two forms of protamine P2. Eur $J$ Biochem 156, 5-8

Pirhonen A, Linnala-Kankunnen $A$, Mäenpää PH (1989) Comparison of partial amino-acid se- quences of two protamine 2 variants from stallion sperm. Febs Lett 244, 199-202

Sautière $P$, Martinage A, Bélaïche D, Arkhis A, Chevaillier $P$ (1988) Comparison of the amino-acid sequences of human protamines HP2 and HP3 and of intermediate basic nuclear proteins HPS1 and HPS2. Structural evidence that HPS1 and HPS2 are proprotamines. J Biol Chem 263, 11059-11062

Yelick PC, Balhorn R, Johnson PA, Corzett M, Mazrimas JA, Kleene KC, Hecht NB (1987) Mouse protamine 2 is synthesized as a precursor whereas mouse protamine 1 is not. Mol Cell Biol 7, 2173-2179 